Supporting Information for

\title{
Single Diastereomer of a Macrolactam Core Binds Specifically to Myeloid Cell Leukemia 1 (MCL1)
}

Chao Fang ${ }^{\dagger *}$, Brendan D’Souza ${ }^{\dagger}$, Christopher F. Thompson ${ }^{\dagger}$, Matthew C. Clifton ${ }^{\dagger \dagger}$, James W. Fairman $^{\dagger \dagger}$, Ben Fulroth ${ }^{\dagger}$, Alison Leed ${ }^{\dagger}$, Patrick McCarren ${ }^{\dagger}$, Lili Wang ${ }^{\dagger}$, Yikai Wang ${ }^{\dagger}$, Clementine Feau $^{\dagger}$, Virendar K. Kaushik ${ }^{\dagger}$, Michelle Palmer ${ }^{\dagger}$, Guo Wei ${ }^{\dagger}$, Todd R. Golub ${ }^{\dagger \#}$, Brian K. Hubbard ${ }^{\dagger}$, and Michael H. Serrano- $\mathrm{Wu}^{\dagger} *$

${ }^{\dagger}$ Broad Institute, 415 Main Street, Cambridge, MA 02142

\# Dana-Farber Cancer Institute and Howard Hughes Medical Institute

${ }^{\dagger}$ Beryllium, 3 Preston Court, Bedford, MA 01730

1. Synthetic procedures and compound characterization SI-2

2. Materials and methods for biological assays

SI-10

3. X-ray information

SI-13

Figure SI-1: $\quad$ DSF screening data for 6 and related analog SI-3

Figure SI-2: $\quad$ The lowest energy conformation of the free ligand 6 (green), 7 (orange), and the $\mathrm{C} 2$ epimer of 6 (magenta) aligned on the macrocycle core in the crystal structure of 7 (yellow)

Figure SI-3: $\quad$ DSC data for all eight isomers of compound 6

Figure SI-4: $\quad$ FP data of 7 against Bcl-2 and Bcl-xL

Figure SI-5: $\quad$ DSC and ITC data of fragments 19-20 


\section{Synthetic procedure and compound characterization:}

General: All oxygen and/or moisture sensitive reactions were carried out under $\mathrm{N}_{2}$ atmosphere in oven or flame dried glassware. All reagents and solvents were purchased from commercial vendors and used as received. NMR spectra were recorded on a Bruker $300 \mathrm{MHz}$ or $400 \mathrm{MHz}$ spectrometer. Proton and carbon chemical shifts are reported in ppm referenced to the NMR solvent. Flash chromatography was performed using 40-60 um silica gel (60 mesh) on a Teledyne Isco Combiflash Rf. Tandem liquid chromatography/Mass Spectrometry (LCMS) was performed on a Waters 2795 separations module and 3100 mass detector, with a Poroshell 120 EC-C18 column. Mobile phase A consisted of $0.01 \%$ formic acid in water, while mobile phase B consisted of $0.01 \%$ formic acid in acetonitrile. The gradient ran from $5 \%$ to $95 \%$ mobile phase B over $2.5 \mathrm{~min}$ at $1.75 \mathrm{~mL} / \mathrm{min}$. Purity was measured by UV absorbance at $210 \mathrm{~nm}$. HRMS was obtained by a hybrid quadrupoleorbitrap mass spectrometer (QExactive, Thermo Scientific) set in positive ion mode.

Synthesis of the library members of the structure SI-1 has been reported previously. ${ }^{1}$

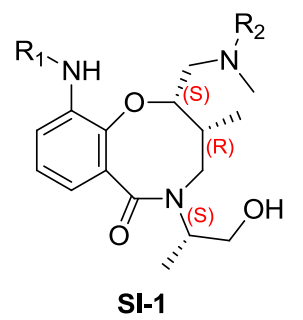




\section{Synthesis of 7:}
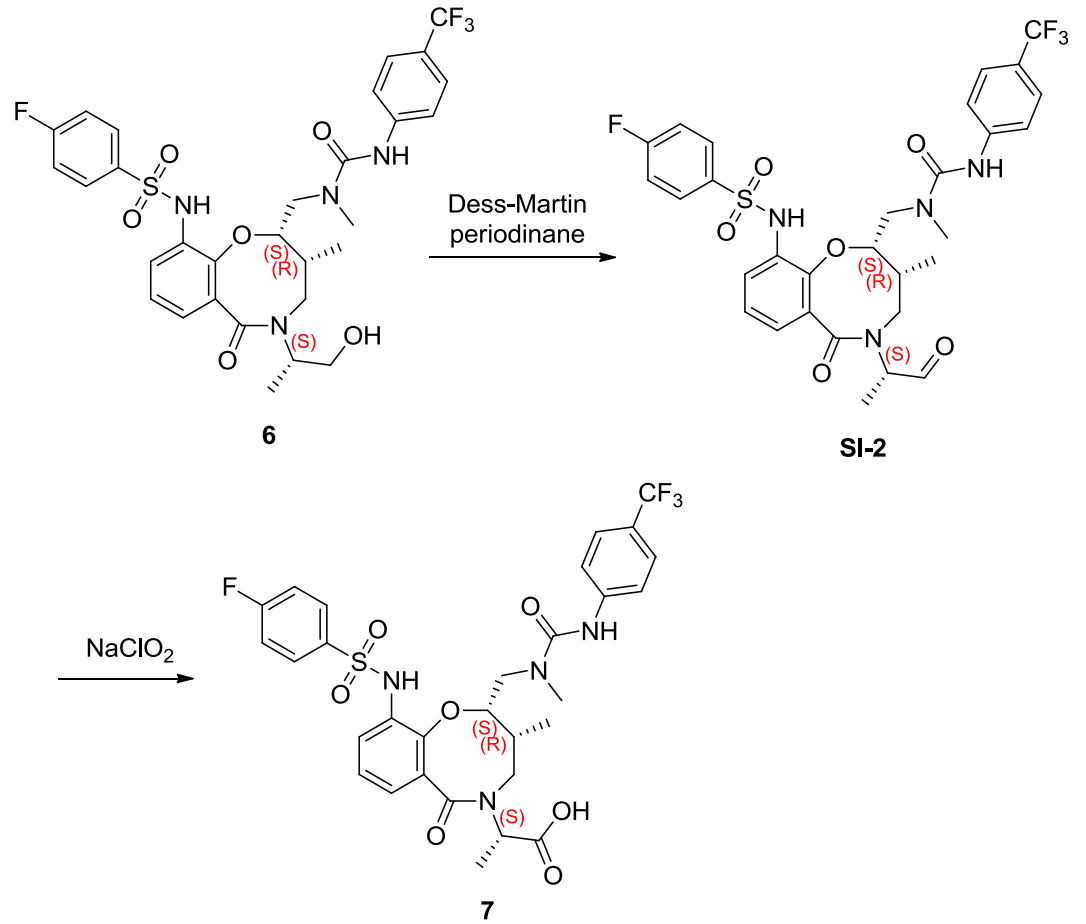

(S)-2-((2S,3R)-10-(4-fluorophenylsulfonamido)-3-methyl-2-((1-methyl-3-(4(trifluoromethyl)phenyl)ureido)methyl)-6-oxo-3,4-dihydro-2H-benzo[b][1,5]oxazocin5(6H)-yl)propanoic acid (7): 6 (47.5 mg, $0.073 \mathrm{mmol})$ was dissolved in DCM (2 mL), and the solution was cooled to $0{ }^{\circ} \mathrm{C}$. Dess-Martin Periodinane $(93 \mathrm{mg}, 0.218 \mathrm{mmol})$ was added and then the ice bath was removed. After $20 \mathrm{~min}$, the reaction mixture was diluted with EtOAc $(50 \mathrm{~mL})$, and washed with $1 \mathrm{~N} \mathrm{NaOH}$ and brine. The organic layer was dried over $\mathrm{Na}_{2} \mathrm{SO}_{4}$, filtered, and concentrated. Column chromotography with 0 to $10 \% \mathrm{MeOH} / \mathrm{DCM}$ eluted the product SI-2 (42 mg, 88\% yield) as colorless oil: ${ }^{1} \mathrm{H}$ NMR (400 MHz, $\left.\mathrm{CDCl}_{3}\right) \delta$ $9.62(\mathrm{~s}, 1 \mathrm{H}), 8.58(\mathrm{~s}, 1 \mathrm{H}), 7.81(\mathrm{dd}, \mathrm{J}=8.8,5.0 \mathrm{~Hz}, 2 \mathrm{H}), 7.74-7.48(\mathrm{~m}, 5 \mathrm{H}), 7.22(\mathrm{~d}, \mathrm{~J}=7.6$ Hz, 1H), 6.93 (dt, J = 27.9, 8.3 Hz, 3H), 6.63 (s, 1H), 4.85 (d, J = 9.6 Hz, 1H), 4.61 (ddd, J = 36.3, 14.9, 8.6 Hz, 2H), 3.32 - 2.97 (m, 5H), $2.82(\mathrm{~d}, \mathrm{~J}=14.5 \mathrm{~Hz}, 1 \mathrm{H}), 1.90(\mathrm{dt}, \mathrm{J}=13.0,6.8$ $\mathrm{Hz}, 1 \mathrm{H}), 1.64-1.40(\mathrm{~m}, 3 \mathrm{H}), 0.71(\mathrm{~d}, \mathrm{~J}=6.6 \mathrm{~Hz}, 3 \mathrm{H})$.

SI-2 $(36 \mathrm{mg}, 0.056 \mathrm{mmol}$ ) was dissolved in t-BuOH (1 mL), 2-methyl-2-butene $(0.25 \mathrm{~mL})$ and acetonitrile $(0.1 \mathrm{~mL})$ and the homogenous solution was treated with $\mathrm{NaClO}_{2}(45.5 \mathrm{mg}$, $0.504 \mathrm{mmol})$ and $\mathrm{NaH}_{2} \mathrm{PO}_{4}(40.3 \mathrm{mg}, 0.336 \mathrm{mmol})$ in Water $(0.5 \mathrm{~mL})$. After $10 \mathrm{~min}$, LCMS indicated the reaction was complete. The reaction mixture was partitioned between EtOAc 
$(50 \mathrm{~mL})$ and $1 \mathrm{~N} \mathrm{HCl}(10 \mathrm{~mL})$, and the organic layer was washed with brine $(2 \times 10 \mathrm{~mL})$, dried over $\mathrm{Na}_{2} \mathrm{SO}_{4}$, filtered, and concentrated. Preparative TLC with $10 \% \mathrm{MeOH} / \mathrm{DCM}$ afforded 7 as white solid (24.2 mg, 65\% yield): ${ }^{1} \mathrm{H}$ NMR (400 MHz, MeOD) $\delta$ 7.63-7.78 (m, 4H), 7.41-7.53 (m, 3H), 7.02-7.13 (m, 3H), 6.82 (m, 1H), 4.87 (d, J = 9.5 Hz, 1H), 4.66 (d, J = 7.7 Hz, 1H), $4.34(\mathrm{dd}, \mathrm{J}=14.5,9.7 \mathrm{~Hz}, 1 \mathrm{H}), 2.96(\mathrm{~s}, 3 \mathrm{H}), 2.90-2.65(\mathrm{~m}, 2 \mathrm{H}), 1.97(\mathrm{~m}$, $1 \mathrm{H}), 1.43(\mathrm{~d}, \mathrm{~J}=7.2 \mathrm{~Hz}, 3 \mathrm{H}), 0.80(\mathrm{~m}, 1 \mathrm{H}), 0.51(\mathrm{~d}, \mathrm{~J}=6.4 \mathrm{~Hz}, 3 \mathrm{H})$. LCMS t $\mathrm{R}_{\mathrm{R}}=1.50 \mathrm{~min}$. HRMS (ESI ${ }^{+}$): calcd for $\mathrm{C}_{30} \mathrm{H}_{30} \mathrm{~F}_{4} \mathrm{~N}_{4} \mathrm{O}_{7} \mathrm{~S}(\mathrm{M}+\mathrm{H})$ : 667.1844; found: 667.1837.

8-18 were prepared using similar procedure as above. Analytical data for 8-18 follows below:

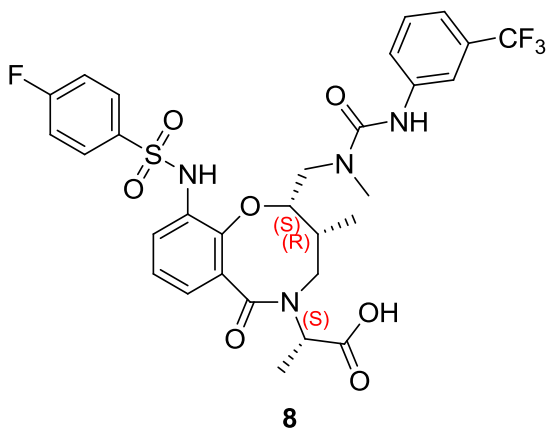

${ }^{1} \mathrm{H}$ NMR (400 MHz, MeOD) $\delta 7.83(\mathrm{~d}, \mathrm{~J}=8.2 \mathrm{~Hz}, 1 \mathrm{H}), 7.75-7.64(\mathrm{~m}, 3 \mathrm{H}), 7.49(\mathrm{~d}, \mathrm{~J}=7.8$ $\mathrm{Hz}, 1 \mathrm{H}), 7.36(\mathrm{t}, \mathrm{J}=8.1 \mathrm{~Hz}, 1 \mathrm{H}), 7.23(\mathrm{dt}, \mathrm{J}=23.4,7.4 \mathrm{~Hz}, 4 \mathrm{H}), 7.10(\mathrm{~d}, \mathrm{~J}=7.9 \mathrm{~Hz}, 1 \mathrm{H})$, $7.03(\mathrm{t}, \mathrm{J}=8.6 \mathrm{~Hz}, 2 \mathrm{H}), 6.80(\mathrm{t}, \mathrm{J}=8.0 \mathrm{~Hz}, 1 \mathrm{H}), 4.94(\mathrm{~d}, \mathrm{~J}=9.6 \mathrm{~Hz}, 1 \mathrm{H}), 4.67$ (q, J = $7.3 \mathrm{~Hz}$, $1 \mathrm{H}), 4.31(\mathrm{dd}, \mathrm{J}=14.4,9.8 \mathrm{~Hz}, 1 \mathrm{H}), 3.21(\mathrm{~s}, 4 \mathrm{H}), 2.95(\mathrm{~s}, 3 \mathrm{H}), 2.84-2.71(\mathrm{~m}, 2 \mathrm{H}), 2.10$ $1.97(\mathrm{~m}, 1 \mathrm{H}), 1.38(\mathrm{~d}, \mathrm{~J}=7.4 \mathrm{~Hz}, 3 \mathrm{H}), 0.47(\mathrm{~d}, \mathrm{~J}=6.6 \mathrm{~Hz}, 3 \mathrm{H}) . \mathrm{LCMS} \mathrm{t}_{\mathrm{R}}=1.49 \mathrm{~min}$. HRMS $\left(\mathrm{ESI}^{+}\right)$: calcd for $\mathrm{C}_{30} \mathrm{H}_{30} \mathrm{~F}_{4} \mathrm{~N}_{4} \mathrm{O}_{7} \mathrm{~S}(\mathrm{M}+\mathrm{H})$ : 667.1844; found: 667.1835 . 


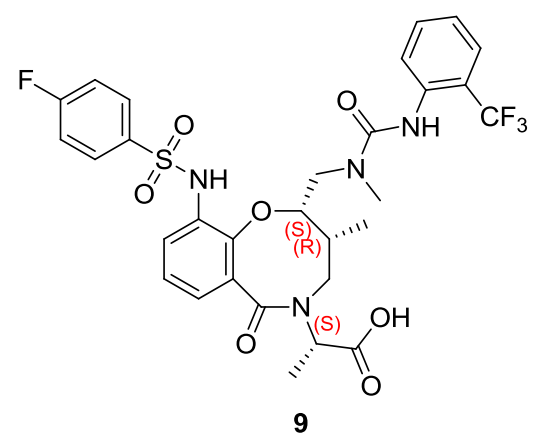

${ }^{1} \mathrm{H}$ NMR (400 MHz, MeOD) $\delta 7.75-7.63(\mathrm{~m}, 3 \mathrm{H}), 7.56(\mathrm{dd}, \mathrm{J}=8.0,4.5 \mathrm{~Hz}, 3 \mathrm{H}), 7.25$ (dq, J $=17.3,9.5,8.6 \mathrm{~Hz}, 3 \mathrm{H}), 7.15(\mathrm{~d}, \mathrm{~J}=8.0 \mathrm{~Hz}, 1 \mathrm{H}), 7.08(\mathrm{q}, \mathrm{J}=8.5,7.4 \mathrm{~Hz}, 2 \mathrm{H}), 6.84(\mathrm{t}, \mathrm{J}=$ $8.0 \mathrm{~Hz}, 1 \mathrm{H}), 4.95(\mathrm{~d}, \mathrm{~J}=9.7 \mathrm{~Hz}, 1 \mathrm{H}), 4.66(\mathrm{q}, \mathrm{J}=7.4 \mathrm{~Hz}, 1 \mathrm{H}), 4.28(\mathrm{dd}, \mathrm{J}=14.4,9.9 \mathrm{~Hz}$, 1H), $3.26-3.12(\mathrm{~m}, 4 \mathrm{H}), 2.95(\mathrm{~s}, 3 \mathrm{H}), 2.82-2.65(\mathrm{~m}, 2 \mathrm{H}), 2.04(\mathrm{td}, \mathrm{J}=13.3,12.1,6.9 \mathrm{~Hz}$, $1 \mathrm{H}), 1.36(\mathrm{~d}, \mathrm{~J}=7.4 \mathrm{~Hz}, 3 \mathrm{H}), 0.39(\mathrm{~d}, \mathrm{~J}=6.6 \mathrm{~Hz}, 3 \mathrm{H})$. LCMS t $\mathrm{R}=1.44 \mathrm{~min}$. HRMS $\left(\mathrm{ESI}^{+}\right)$: calcd for $\mathrm{C}_{30} \mathrm{H}_{30} \mathrm{~F}_{4} \mathrm{~N}_{4} \mathrm{O}_{7} \mathrm{~S}(\mathrm{M}+\mathrm{H})$ : 667.1844; found: 667.1835 .

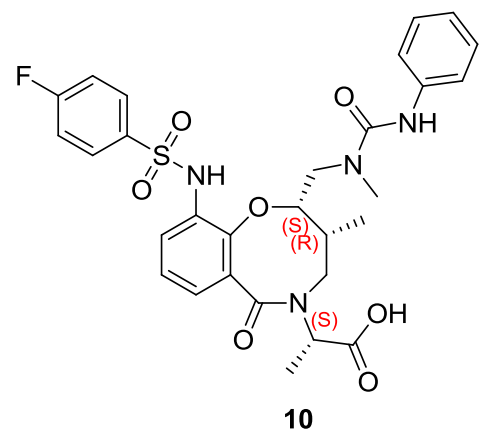

${ }^{1} \mathrm{H}$ NMR (400 MHz, $\left.\mathrm{CDCl}_{3}\right) \delta 8.95(\mathrm{~s}, 2 \mathrm{H}), 8.47(\mathrm{~s}, 1 \mathrm{H}), 7.77(\mathrm{dd}, \mathrm{J}=8.3,4.7 \mathrm{~Hz}, 2 \mathrm{H}), 7.51$ $(\mathrm{d}, \mathrm{J}=7.8 \mathrm{~Hz}, 2 \mathrm{H}), 7.42(\mathrm{~d}, \mathrm{~J}=8.1 \mathrm{~Hz}, 1 \mathrm{H}), 7.27(\mathrm{q}, \mathrm{J}=7.9,6.3 \mathrm{~Hz}, 3 \mathrm{H}), 7.18(\mathrm{dd}, \mathrm{J}=10.9$, $6.7 \mathrm{~Hz}, 2 \mathrm{H}), 7.15-6.97(\mathrm{~m}, 3 \mathrm{H}), 6.91(\mathrm{t}, \mathrm{J}=8.5 \mathrm{~Hz}, 2 \mathrm{H}), 6.79(\mathrm{t}, \mathrm{J}=8.1 \mathrm{~Hz}, 1 \mathrm{H}), 4.96(\mathrm{q}, \mathrm{J}$ $=7.2 \mathrm{~Hz}, 1 \mathrm{H}), 4.76(\mathrm{~d}, \mathrm{~J}=9.2 \mathrm{~Hz}, 1 \mathrm{H}), 4.44(\mathrm{dd}, \mathrm{J}=14.6,9.3 \mathrm{~Hz}, 1 \mathrm{H}), 3.27-2.98(\mathrm{~m}, 2 \mathrm{H})$, $2.86(\mathrm{~s}, 3 \mathrm{H}), 2.68(\mathrm{~d}, \mathrm{~J}=14.5 \mathrm{~Hz}, 1 \mathrm{H}), 2.38(\mathrm{~s}, 1 \mathrm{H}), 1.89(\mathrm{dt}, \mathrm{J}=13.8,7.1 \mathrm{~Hz}, 1 \mathrm{H}), 1.50(\mathrm{~s}$, $3 \mathrm{H}), 0.63(\mathrm{~d}, \mathrm{~J}=6.5 \mathrm{~Hz}, 3 \mathrm{H})$. LCMS $\mathrm{t}_{\mathrm{R}}=1.35 \mathrm{~min}$. HRMS $\left(\mathrm{ESI}^{+}\right)$: calcd for $\mathrm{C}_{29} \mathrm{H}_{31} \mathrm{~F}_{4} \mathrm{~N}_{4} \mathrm{O}_{7} \mathrm{~S}$ (M+H): 599.1970; found: 599.1965. 


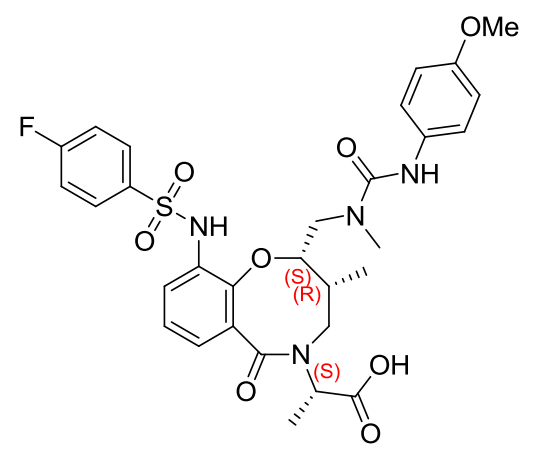

11

${ }^{1} \mathrm{H}$ NMR (400 MHz, DMSO-d6) $\delta 12.63(\mathrm{~s}, 1 \mathrm{H}), 9.09$ (s, 1H), 8.16 (s, 1H), 7.73 (dd, J = 8.7, $4.9 \mathrm{~Hz}, 2 \mathrm{H}), 7.43$ (dd, J = 8.6, 4.3 Hz, 3H), 7.30 (t, J = 8.6 Hz, 2H), $7.17-7.05(\mathrm{~m}, 1 \mathrm{H}), 6.99$ - $6.87(\mathrm{~m}, 1 \mathrm{H}), 6.86-6.78(\mathrm{~m}, 2 \mathrm{H}), 4.68(\mathrm{~d}, \mathrm{~J}=8.5 \mathrm{~Hz}, 1 \mathrm{H}), 4.49(\mathrm{q}, \mathrm{J}=7.0 \mathrm{~Hz}, 1 \mathrm{H}), 4.11$ $(\mathrm{dt}, \mathrm{J}=14.8,6.6 \mathrm{~Hz}, 1 \mathrm{H}), 3.71(\mathrm{~d}, \mathrm{~J}=3.6 \mathrm{~Hz}, 3 \mathrm{H}), 3.30-3.22(\mathrm{~m}, 1 \mathrm{H}), 3.17(\mathrm{~s}, 1 \mathrm{H}), 2.99(\mathrm{~d}$, $\mathrm{J}=13.7 \mathrm{~Hz}, 1 \mathrm{H}), 2.93(\mathrm{~s}, 3 \mathrm{H}), 2.85-2.72(\mathrm{~m}, 2 \mathrm{H}), 1.98(\mathrm{~s}, 1 \mathrm{H}), 1.42(\mathrm{~d}, \mathrm{~J}=7.1 \mathrm{~Hz}, 3 \mathrm{H})$, $0.54(\mathrm{~d}, \mathrm{~J}=6.5 \mathrm{~Hz}, 3 \mathrm{H})$. LCMS $\mathrm{t}_{\mathrm{R}}=1.33 \mathrm{~min}$. HRMS $\left(\mathrm{ESI}^{+}\right)$: calcd for $\mathrm{C}_{30} \mathrm{H}_{33} \mathrm{FN}_{4} \mathrm{O}_{8} \mathrm{~S}$ $(\mathrm{M}+\mathrm{H}): 629.2076$; found: 629.2071 .

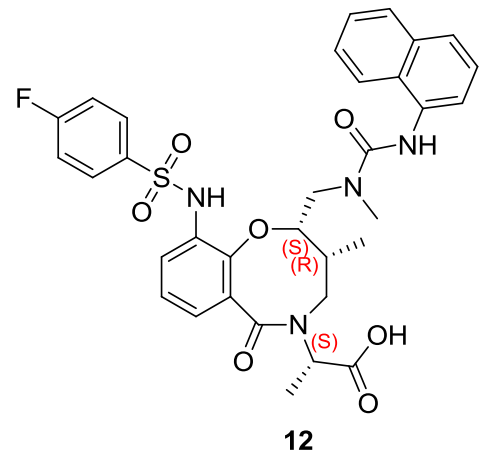

${ }^{1} \mathrm{H}$ NMR $\left(400 \mathrm{MHz}, \mathrm{CDCl}_{3}\right) \delta 9.61(\mathrm{~s}, 2 \mathrm{H}), 8.17(\mathrm{~s}, 1 \mathrm{H}), 7.85(\mathrm{~d}, \mathrm{~J}=8.1 \mathrm{~Hz}, 1 \mathrm{H}), 7.75-7.55$ (m, 5H), $7.41(\mathrm{dq}, \mathrm{J}=40.4,7.7 \mathrm{~Hz}, 5 \mathrm{H}), 7.27(\mathrm{~s}, 2 \mathrm{H}), 6.93(\mathrm{~d}, \mathrm{~J}=7.8 \mathrm{~Hz}, 1 \mathrm{H}), 6.83$ (t, J = $8.3 \mathrm{~Hz}, 2 \mathrm{H}), 6.60(\mathrm{t}, \mathrm{J}=7.8 \mathrm{~Hz}, 1 \mathrm{H}), 4.95-4.73(\mathrm{~m}, 2 \mathrm{H}), 4.22(\mathrm{t}, \mathrm{J}=11.5 \mathrm{~Hz}, 1 \mathrm{H}), 3.10(\mathrm{dd}$, $\mathrm{J}=15.5,4.8 \mathrm{~Hz}, 1 \mathrm{H}), 2.95(\mathrm{~d}, \mathrm{~J}=14.7 \mathrm{~Hz}, 4 \mathrm{H}), 2.81(\mathrm{~d}, \mathrm{~J}=14.1 \mathrm{~Hz}, 1 \mathrm{H}), 1.87(\mathrm{q}, \mathrm{J}=9.0$, $6.0 \mathrm{~Hz}, 1 \mathrm{H}), 1.41(\mathrm{~s}, 3 \mathrm{H}), 0.60(\mathrm{~d}, \mathrm{~J}=6.5 \mathrm{~Hz}, 3 \mathrm{H}) . \mathrm{LCMS} \mathrm{t}_{\mathrm{R}}=1.39 \mathrm{~min}$. HRMS $\left(\mathrm{ESI}^{+}\right)$: calcd for $\mathrm{C}_{33} \mathrm{H}_{33} \mathrm{FN}_{4} \mathrm{O}_{7} \mathrm{~S}(\mathrm{M}+\mathrm{H})$ : 649.2127; found: 649.2125. 


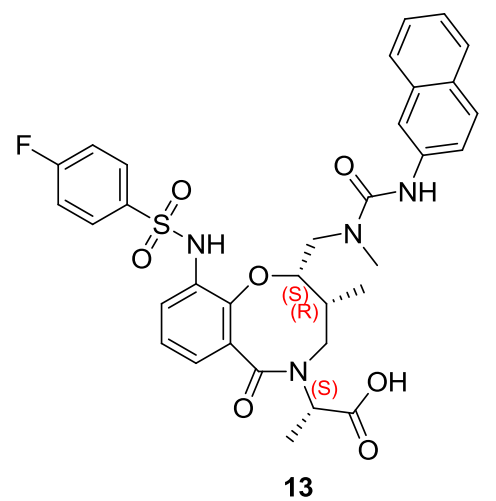

${ }^{1} \mathrm{H}$ NMR (400 MHz, MeOD) $\delta 8.17(\mathrm{~s}, 1 \mathrm{H}), 7.77(\mathrm{~d}, \mathrm{~J}=8.1 \mathrm{~Hz}, 1 \mathrm{H}), 7.75-7.64(\mathrm{~m}, 4 \mathrm{H})$, $7.48(\mathrm{dd}, \mathrm{J}=13.1,8.4 \mathrm{~Hz}, 2 \mathrm{H}), 7.32(\mathrm{t}, \mathrm{J}=7.5 \mathrm{~Hz}, 1 \mathrm{H}), 7.29-7.19(\mathrm{~m}, 4 \mathrm{H}), 7.11(\mathrm{~d}, \mathrm{~J}=7.9$ Hz, 1H), $6.97(\mathrm{t}, \mathrm{J}=8.6 \mathrm{~Hz}, 2 \mathrm{H}), 6.82(\mathrm{t}, \mathrm{J}=8.0 \mathrm{~Hz}, 1 \mathrm{H}), 4.88(\mathrm{~d}, \mathrm{~J}=9.7 \mathrm{~Hz}, 1 \mathrm{H}), 4.81(\mathrm{~s}$, 1H), $4.67(\mathrm{~d}, \mathrm{~J}=7.6 \mathrm{~Hz}, 1 \mathrm{H}), 4.41(\mathrm{dd}, \mathrm{J}=14.6,9.9 \mathrm{~Hz}, 1 \mathrm{H}), 2.98(\mathrm{~s}, 3 \mathrm{H}), 2.86(\mathrm{t}, \mathrm{J}=14.2$ $\mathrm{Hz}, 2 \mathrm{H}), 2.80-2.71(\mathrm{~m}, 2 \mathrm{H}), 2.04-1.91(\mathrm{~m}, 2 \mathrm{H}), 1.45(\mathrm{~d}, \mathrm{~J}=7.4 \mathrm{~Hz}, 3 \mathrm{H}), 0.53$ (d, J = 6.5 $\mathrm{Hz}, 3 \mathrm{H})$. LCMS $\mathrm{t}_{\mathrm{R}}=1.49$ min. HRMS $\left(\mathrm{ESI}^{+}\right)$: calcd for $\mathrm{C}_{33} \mathrm{H}_{33} \mathrm{FN}_{4} \mathrm{O}_{7} \mathrm{~S}(\mathrm{M}+\mathrm{H}): 649.2127$; found: 649.2126 .

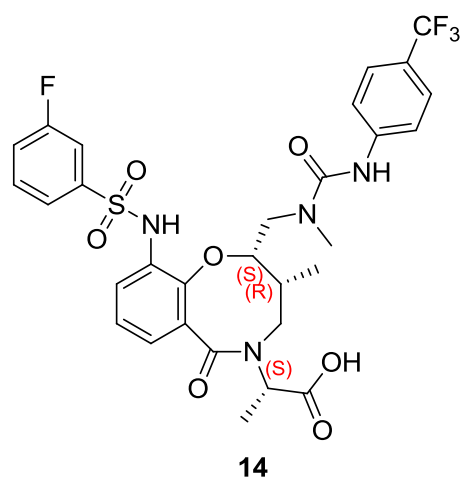

${ }^{1} \mathrm{H}$ NMR (400 MHz, MeOD) $\delta 7.80(\mathrm{~d}, \mathrm{~J}=8.4 \mathrm{~Hz}, 2 \mathrm{H}), 7.63(\mathrm{~d}, \mathrm{~J}=7.5 \mathrm{~Hz}, 1 \mathrm{H}), 7.56(\mathrm{~d}, \mathrm{~J}=$ $7.6 \mathrm{~Hz}, 4 \mathrm{H}), 7.47(\mathrm{td}, \mathrm{J}=8.0,4.7 \mathrm{~Hz}, 1 \mathrm{H}), 7.34(\mathrm{pd}, \mathrm{J}=8.4,5.5,3.3 \mathrm{~Hz}, 1 \mathrm{H}), 7.23(\mathrm{t}, \mathrm{J}=9.4$ Hz, 1H), $7.19-7.09$ (m, 1H), $6.95(\mathrm{t}, \mathrm{J}=8.0 \mathrm{~Hz}, 1 \mathrm{H}), 4.78(\mathrm{q}, \mathrm{J}=7.4 \mathrm{~Hz}, 1 \mathrm{H}), 4.47$ (dd, J = 14.5, $9.8 \mathrm{~Hz}, 1 \mathrm{H}), 3.07$ (s, 3H), $2.99-2.81(\mathrm{~m}, 2 \mathrm{H}), 2.34(\mathrm{~s}, 1 \mathrm{H}), 2.08(\mathrm{dt}, \mathrm{J}=12.6,6.4 \mathrm{~Hz}$, $1 \mathrm{H}), 1.55(\mathrm{~d}, \mathrm{~J}=7.4 \mathrm{~Hz}, 3 \mathrm{H}), 0.61(\mathrm{~d}, \mathrm{~J}=6.6 \mathrm{~Hz}, 3 \mathrm{H})$. LCMS t $t_{\mathrm{R}}=1.50 \mathrm{~min}$. HRMS $\left(\mathrm{ESI}^{+}\right)$: calcd for $\mathrm{C}_{30} \mathrm{H}_{30} \mathrm{~F}_{4} \mathrm{~N}_{4} \mathrm{O}_{7} \mathrm{~S}(\mathrm{M}+\mathrm{H})$ : 667.1844; found: 667.1843. 


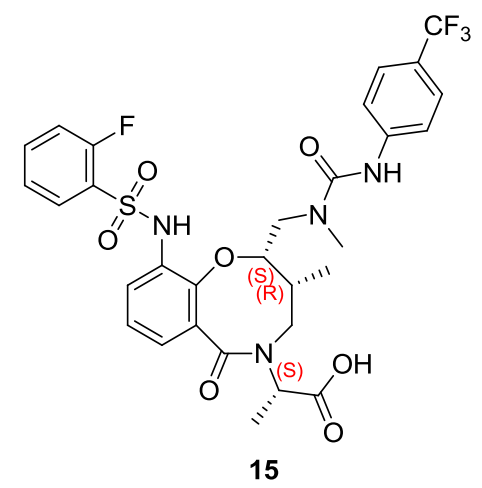

${ }^{1} \mathrm{H}$ NMR (400 MHz, MeOD) $\delta 7.75(\mathrm{t}, \mathrm{J}=7.7 \mathrm{~Hz}, 1 \mathrm{H}), 7.65(\mathrm{~d}, \mathrm{~J}=8.3 \mathrm{~Hz}, 2 \mathrm{H}), 7.53-7.37$ (m, 4H), 7.29 - $7.19(\mathrm{~m}, 1 \mathrm{H}), 7.10(\mathrm{ddt}, \mathrm{J}=21.5,13.8,7.7 \mathrm{~Hz}, 4 \mathrm{H}), 6.76(\mathrm{t}, \mathrm{J}=7.9 \mathrm{~Hz}, 1 \mathrm{H})$, $4.91(\mathrm{~d}, \mathrm{~J}=9.5 \mathrm{~Hz}, 1 \mathrm{H}), 4.70-4.61(\mathrm{~m}, 1 \mathrm{H}), 4.40(\mathrm{dd}, \mathrm{J}=14.5,9.7 \mathrm{~Hz}, 1 \mathrm{H}), 3.27(\mathrm{~d}, \mathrm{~J}=4.6$ $\mathrm{Hz}, 0 \mathrm{H}), 3.21$ (s, 5H), 2.97 (s, 4H), $2.91-2.73$ (m, 2H), 2.22 (s, 1H), 2.03 (dt, J = 12.8, 6.1 $\mathrm{Hz}, 1 \mathrm{H}), 1.45(\mathrm{~d}, \mathrm{~J}=7.4 \mathrm{~Hz}, 3 \mathrm{H}), 0.71(\mathrm{~d}, \mathrm{~J}=6.6 \mathrm{~Hz}, 3 \mathrm{H})$. LCMS $\mathrm{t}_{\mathrm{R}}=1.46 \mathrm{~min}$. HRMS $\left(\mathrm{ESI}^{+}\right)$: calcd for $\mathrm{C}_{30} \mathrm{H}_{30} \mathrm{~F}_{4} \mathrm{~N}_{4} \mathrm{O}_{7} \mathrm{~S}(\mathrm{M}+\mathrm{H})$ : 667.1844; found: 667.1843 .

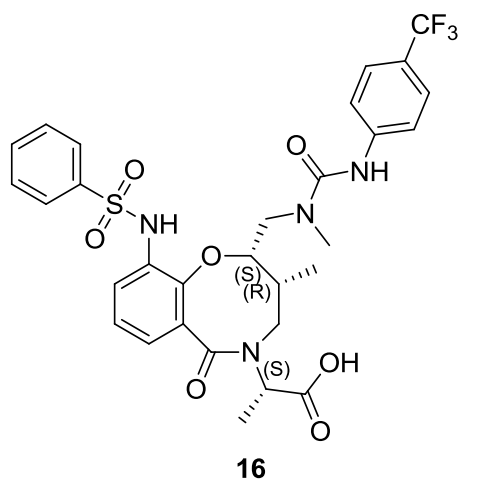

${ }^{1} \mathrm{H}$ NMR (400 MHz, MeOD) $\delta 7.79(\mathrm{t}, \mathrm{J}=9.0 \mathrm{~Hz}, 4 \mathrm{H}), 7.62(\mathrm{~d}, \mathrm{~J}=7.6 \mathrm{~Hz}, 1 \mathrm{H}), 7.56(\mathrm{~d}, \mathrm{~J}=$ $8.3 \mathrm{~Hz}, 3 \mathrm{H}), 7.43$ (t, J = 7.7 Hz, 2H), $7.27-7.09$ (m, 3H), 6.92 (t, J = 7.9 Hz, 1H), 4.76 (q, J $=7.3 \mathrm{~Hz}, 1 \mathrm{H}), 4.47(\mathrm{dd}, \mathrm{J}=14.5,9.8 \mathrm{~Hz}, 1 \mathrm{H}), 3.39-3.23(\mathrm{~m}, 3 \mathrm{H}), 3.06(\mathrm{~s}, 3 \mathrm{H}), 2.97-2.79$ (m, 2H), $2.33(\mathrm{~s}, 1 \mathrm{H}), 2.06(\mathrm{dq}, \mathrm{J}=12.3,6.2 \mathrm{~Hz}, 1 \mathrm{H}), 1.54(\mathrm{~d}, \mathrm{~J}=7.4 \mathrm{~Hz}, 3 \mathrm{H}), 0.60(\mathrm{~d}, \mathrm{~J}=$ 6.6 Hz, 3H). LCMS $t_{R}=1.46$ min. HRMS $\left(\mathrm{ESI}^{+}\right)$: calcd for $\mathrm{C}_{30} \mathrm{H}_{31} \mathrm{~F}_{3} \mathrm{~N}_{4} \mathrm{O}_{7} \mathrm{~S}(\mathrm{M}+\mathrm{H})$ : 649.1938; found: 649.1939 . 


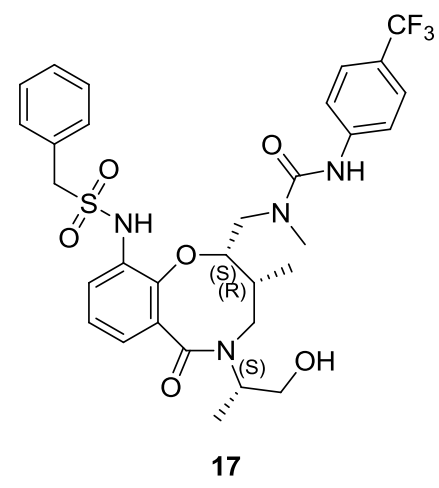

${ }^{1} \mathrm{H}$ NMR (400 MHz, MeOD) $\delta 7.70(\mathrm{~d}, \mathrm{~J}=8.4 \mathrm{~Hz}, 2 \mathrm{H}), 7.52(\mathrm{~d}, \mathrm{~J}=8.4 \mathrm{~Hz}, 2 \mathrm{H}), 7.40-7.08$ $(\mathrm{m}, 7 \mathrm{H}), 6.85(\mathrm{t}, \mathrm{J}=8.0 \mathrm{~Hz}, 1 \mathrm{H}), 5.02(\mathrm{~d}, \mathrm{~J}=9.5 \mathrm{~Hz}, 1 \mathrm{H}), 4.76(\mathrm{q}, \mathrm{J}=7.2 \mathrm{~Hz}, 1 \mathrm{H}), 4.50$ (dd, $\mathrm{J}=14.5,9.7 \mathrm{~Hz}, 1 \mathrm{H}), 4.38-4.17(\mathrm{~m}, 2 \mathrm{H}), 3.76(\mathrm{~d}, \mathrm{~J}=6.8 \mathrm{~Hz}, 2 \mathrm{H}), 3.48(\mathrm{dd}, \mathrm{J}=15.4,5.7$ $\mathrm{Hz}, 1 \mathrm{H}), 3.27$ - 3.14 (m, 1H), 3.05-3.15 (m, 4H), 2.26 (m, 1H), 1.25 (d, J = 6.8 Hz, 3H), 1.02 $(\mathrm{d}, \mathrm{J}=6.5 \mathrm{~Hz}, 3 \mathrm{H})$. LCMS $\mathrm{t}_{\mathrm{R}}=1.45 \mathrm{~min}$. HRMS $\left(\mathrm{ESI}^{+}\right)$: calcd for $\mathrm{C}_{31} \mathrm{H}_{35} \mathrm{~F}_{3} \mathrm{~N}_{4} \mathrm{O}_{6} \mathrm{~S}(\mathrm{M}+\mathrm{H})$ : 649.2302; found: 649.2302 .

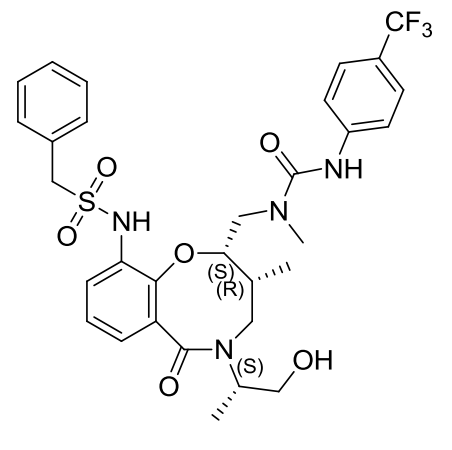

18

${ }^{1} \mathrm{H}$ NMR $(400 \mathrm{MHz}, \mathrm{MeOD}) \delta 7.61(\mathrm{~d}, \mathrm{~J}=8.2 \mathrm{~Hz}, 2 \mathrm{H}), 7.47(\mathrm{~d}, \mathrm{~J}=7.7 \mathrm{~Hz}, 1 \mathrm{H}), 7.38(\mathrm{~d}, \mathrm{~J}=$ $8.5 \mathrm{~Hz}, 2 \mathrm{H}), 7.04-7.14(\mathrm{~m}, 4 \mathrm{H}), 6.94-6.79(\mathrm{~m}, 3 \mathrm{H}), 4.91(\mathrm{~d}, \mathrm{~J}=9.6 \mathrm{~Hz}, 1 \mathrm{H}), 4.66(\mathrm{q}, \mathrm{J}=6.8$ $\mathrm{Hz}, 1 \mathrm{H}), 4.43(\mathrm{~m}, 1 \mathrm{H}), 3.63(\mathrm{~d}, \mathrm{~J}=6.9 \mathrm{~Hz}, 2 \mathrm{H}), 2.75-3.40(\mathrm{~m}, 10 \mathrm{H}), 2.12(\mathrm{~m}, 1 \mathrm{H}), 1.12(\mathrm{~d}, \mathrm{~J}$ $=6.9 \mathrm{~Hz}, 3 \mathrm{H}), 0.84(\mathrm{~d}, \mathrm{~J}=6.6 \mathrm{~Hz}, 3 \mathrm{H}) . \mathrm{LCMS} \mathrm{t}_{\mathrm{R}}=1.52 \mathrm{~min}$. HRMS $\left(\mathrm{ESI}^{+}\right)$: calcd for $\mathrm{C}_{32} \mathrm{H}_{37} \mathrm{~F}_{3} \mathrm{~N}_{4} \mathrm{O}_{6} \mathrm{~S}(\mathrm{M}+\mathrm{H})$ : 663.2459; found: 663.2459 . 


\section{Materials and methods for biological assays:}

a) Differential Scanning Calorimetry (DSC): Human MCL1 (173-329) protein was dialyzed overnight at $4{ }^{\circ} \mathrm{C}$ into $25 \mathrm{mM}$ HEPES buffer, $\mathrm{pH} 7.4,100 \mathrm{mM} \mathrm{NaCl}$, and $0.1 \mathrm{mM}$ TCEP for all DSC experiments. Experiments were conducted on a MicroCal VP-Capillary DSC instrument (Malvern Instruments Ltd.). Each run consisted of a reference cell containing $400 \mu \mathrm{L}$ of $250 \mu \mathrm{M}$ compound in dialysis buffer with $4 \%$ DMSO and a protein cell containing $400 \mu \mathrm{L}$ of $0.5 \mathrm{mg} / \mathrm{mL}$ MCL1 $(\sim 25 \mu \mathrm{M})$ and $250 \mu \mathrm{M}$ compound in dialysis buffer with 4\% DMSO. Melting curves were acquired at a scan rate of $200{ }^{\circ} \mathrm{C} /$ hour, between 35 $100{ }^{\circ} \mathrm{C}$. Buffer scans were subtracted from all runs and analyzed in Origin software, version 7.0552. $\Delta \mathrm{T}_{\mathrm{m}}$ values were determined by subtracting an average $\mathrm{T}_{\mathrm{m}}$ value determined for runs containing apo MCL1 protein from the MCL1 $\mathrm{T}_{\mathrm{m}}$ in the presence of compound.

b) Isothermal Titration Calorimetry (ITC): Human MCL1 (173-329) protein was dialyzed overnight at $4{ }^{\circ} \mathrm{C}$ into $25 \mathrm{mM}$ HEPES buffer, $\mathrm{pH} 7.4,100 \mathrm{mM} \mathrm{NaCl}$, and $0.1 \mathrm{mM}$ TCEP for all ITC experiments. Compounds were prepared in this dialysis buffer at $400 \mu \mathrm{M}$ or $1200 \mu \mathrm{M}$ (fragments) with $4 \%$ DMSO added. $40 \mu \mathrm{L}$ of compound was titrated into $200 \mu \mathrm{L}$ of 25mM MCL1, also in dialysis buffer containing 4\% DMSO. Reference experiments (titration of compound into buffer alone) were subtracted to account for compound heat of dilution effects. Experiments were conducted at $25{ }^{\circ} \mathrm{C}$ with a MicroCal Auto-ITC200 instrument (Malvern Instruments Ltd), using a $1000 \mathrm{rpm}$ stirring speed and a reference power of 10 $\mu \mathrm{cal} / \mathrm{sec}$. ITC curves were analyzed using Origin software, version 7.0552, and the binding affinity $\left(K_{D}\right)$, enthalpy $(\Delta H)$, and entropy $(\Delta S)$ were calculated for each compound.

c) ${ }^{19}$ F NMR: MCL1 protein was dialyzed overnight at $4{ }^{\circ} \mathrm{C}$ into $50 \mathrm{mM}$ HEPES buffer containing $100 \mathrm{mM} \mathrm{NaCl}, 0.1 \mathrm{mM}$ TCEP, 0.0025\% TFA (adjusted to $\mathrm{pH} 7.4$ ). Reference samples of ligand alone were prepared with $50 \mathrm{uL} \mathrm{D} \mathrm{D}_{2} \mathrm{O}, 430 \mathrm{uL}$ of dialysis buffer, and $20 \mathrm{uL}$ of compound (dissolved in DMSO-d6) to afford a final ligand concentration of 60 or $120 \mathrm{uM}$. The final concentration or DMSO-d6 in all samples was $4 \% \mathrm{v} / \mathrm{v}$. Identical samples were prepared with MCL1 protein to yield a final protein concentration of $10 \mathrm{uM}$.

${ }^{19} \mathrm{~F}$ NMR spectra were acquired with ${ }^{1} \mathrm{H}$ decoupling on a $400 \mathrm{MHz}$ Bruker console, temperature $=300 \mathrm{~K}$, relaxation delay $=1 \mathrm{sec}$, 256 scans. With TFA reference set to -76.500 
ppm, chemical shifts were recorded for the CF3 resonance. The chemical shift $\Delta v$ was calculated using the equation below:

$\Delta v=(v 2-v 1) * 376.32(\mathrm{~Hz})$

(Eq. 1)

Using Eq. 1, the following chemical shifts were measured for ligand 7:

\begin{tabular}{c|c|c|c|c}
\hline $\begin{array}{c}\text { Ligand } \\
\text { concentration } \\
(\mathrm{uM})\end{array}$ & $\begin{array}{c}\delta(\mathrm{ppm}) \text { with } \\
\text { protein }\end{array}$ & $\begin{array}{c}\delta(\mathrm{ppm}) \\
\text { without } \\
\text { protein }\end{array}$ & $\Delta v$ & $1 / \Delta v$ \\
\hline 60 & -62.5739 & -62.5882 & 5.3768 & 0.18598423 \\
\hline 120 & -62.5801 & -62.5876 & 2.8200 & 0.35450993 \\
\hline
\end{tabular}

Kd was then calculated based on linear fit using Eq. 2 below:

$[\mathrm{L}]_{0}=[\mathrm{P}]_{0} \Delta_{\max } / \Delta_{\mathrm{obs}}-\mathrm{K}_{\mathrm{d}}$

(Eq. 2)

d) Fluorescence Polarization: A competitive fluorescence polarization (FP) assay was used to determine $\mathrm{IC}_{50}$ values for compounds that bind the recombinant MCL1 (173-329) BH3 groove and displace Tetramethylrhodamine (TAMRA)- labeled Noxa peptide (TAMRAGELEVEFATQLRRFGDKLNF-amide; 21 ${ }^{\text {st }}$ Century Biochemical, Marlborough, MA). Compound stocks were prepared at $10 \mathrm{mM}$ in $100 \%$ DMSO and pipetted into column 1 of a 384 well "compound plate" (Thermo Scientific, Cat \# AB-1056). Compounds were then serially diluted in DMSO starting at $10 \mathrm{mM}$ using a Janus Automated Work Station (Perkin Elmer, Waltham, MA). In addition, DMSO and $1 \mathrm{mM}$ unlabeled Noxa peptide in DMSO were added to each well of columns 11 and 12 respectively. DMSO was used as a negative control, while $1 \mathrm{mM}$ (5 $\mu \mathrm{M}$ final) of unlabeled Noxa peptide was used as a positive control. Next, MCL1 recombinant protein was prepared in buffer $(25 \mathrm{mM}$ Hepes, $100 \mathrm{mM} \mathrm{NaCl}$, $0.005 \%$ Tween 20, 3\% DMSO, pH 7.4) at $400 \mathrm{nM}(2 \mathrm{X})$ and $10 \mu \mathrm{L}$ was added to a 384 well black "assay plate" (Corning, Cat \# 3573). The assay plate was centrifuged for 1 min at 800 RPM, and $100 \mathrm{~nL}$ of serially diluted compound was delivered from the compound plate to the assay plate using a 384W $100 \mathrm{~nL}$ Pin Tool (V \& P Scientific; San Diego, CA). The assay plate was incubated at room temperature for 30 minutes. After incubation, $25 \mathrm{nM}$ TAMRAlabeled Noxa peptide was prepared in buffer (25 mM Hepes, $100 \mathrm{mM} \mathrm{NaCl}, 0.005 \%$ Tween $20, \mathrm{pH} 7.4)$ at $50 \mathrm{nM}(2 \mathrm{X})$ and $10 \mu \mathrm{L}$ added to each well of the assay plate. The assay plate 
was centrifuged for $1 \mathrm{~min}$ at $800 \mathrm{RPM}$, and then incubated at room temperature for 30 minutes. The fluorescence polarization (in $\mathrm{mP}$ units) was measured at room temperature with excitation wavelength of $544 \mathrm{~nm}$ and emission wavelength of $579 \mathrm{~nm}$ using an Envision plate reader (Perkin Elmer, Waltham, MA). Each compound was assayed in duplicates run on two separate assay plates. The percentage inhibition was calculated by $\%$ inhibition $=$ $\left(\frac{\left(\left\{\bar{X}_{\text {min }}\right\}-\{\text { Sample }\}\right)}{\left(\bar{X}_{\text {min }}-X_{\text {high }}\right)}\right) * 100$

Where:

$\begin{array}{cl}\bar{X}_{\text {min }} & \text { Average High mP Signal (DMSO; wells A11 - P11 \& A23 - } \\ \{\text { Sample }\} & \text { Raw mP value } \\ & \text { Average Low mP Signal }(5 \mu \mathrm{M} \text { unlabeled Noxa; wells A12 - } \\ \bar{X}_{\text {high }} & \text { P12 \& A24 - P24) }\end{array}$

Based on percentage inhibition, the $\mathrm{IC}_{50}$ (inhibitor concentration at which $50 \%$ of bound TAMRA-labeled Noxa peptide is displaced) was obtained by fitting the inhibition data using a Levenberg-Marquardt curve-fitting algorithm, with finite difference gradients (Dotmatics, San Diego, CA). 
3. X-ray information: Crystals were grown in the presence of $0.5 \mathrm{mM} 7$ and flash frozen using liquid nitrogen. Data were collected at $100 \mathrm{~K}$ on a Rigaku FR-E+ SuperBright rotating anode generator with VariMax optics and a Saturn 944 detector. All data were reduced with XDS/XSCALE. ${ }^{2}$ The structure was phased by molecular replacement using Phaser from the CCP4 suite of programs with PDB ID 1ANF used as a search model. ${ }^{3,4}$ The structure was completed using multiple cycles of refinement in Phenix ${ }^{5}$ followed by manual rebuilding of the structure using Coot. ${ }^{6}$ The structure was quality checked by Molprobity. ${ }^{7}$ The structure has been deposited in the Protein Data Bank (PDB ID 4WGI).

Table SI-1: Structure refinement statistics

\begin{tabular}{|c|c|}
\hline Dataset & 7 \\
\hline $\mathrm{a}(\AA)$ & 99.30 \\
\hline $\mathrm{b}(\AA)$ & 135.87 \\
\hline$c(\AA)$ & 37.69 \\
\hline$\alpha, \beta, \gamma$ & $90,90,90$ \\
\hline Space group & $\mathrm{P} 2{ }_{1} 2_{1} 2$ \\
\hline Wavelength $(\AA)$ & 1.54 \\
\hline Resolution limit $(\AA)$ & $50-1.85(1.9-1.85)$ \\
\hline Number of observations & $44464(3279)^{*}$ \\
\hline Completeness (\%) & $97.7(99.9)^{*}$ \\
\hline Rmerge (\%) & $0.062(0.508)^{*}$ \\
\hline Mean I/ $\sigma \mathrm{I}$ & $16.2(2.12)^{*}$ \\
\hline \multicolumn{2}{|c|}{ Refinement } \\
\hline Resolution Range $(\AA)$ & $50-1.85$ \\
\hline Rcryst & 0.172 \\
\hline Rfree & 0.219 \\
\hline Mean B-factor $(\AA)$ & 23.43 \\
\hline Rmsd bond lengths $(\AA)$ & 0.007 \\
\hline Rmsd bong angles $\left({ }^{\circ}\right)$ & 1.030 \\
\hline \multicolumn{2}{|c|}{ Ramachandran statistics (\%) } \\
\hline Favored & 98.24 \\
\hline
\end{tabular}




\begin{tabular}{|c|c|}
\hline Allowed & 1.56 \\
\hline Outlier & 0.2 \\
\hline Molprobity score & $0.97\left(100^{\text {th }}\right.$ \\
& percentile $)$ \\
\hline
\end{tabular}

* Highest resolution shell shown in parentheses 
Figure SI-1: DSF screening data for 6 and related analog SI-3 (red: binding hit, blue: nonbinding, yellow: inconclusive).

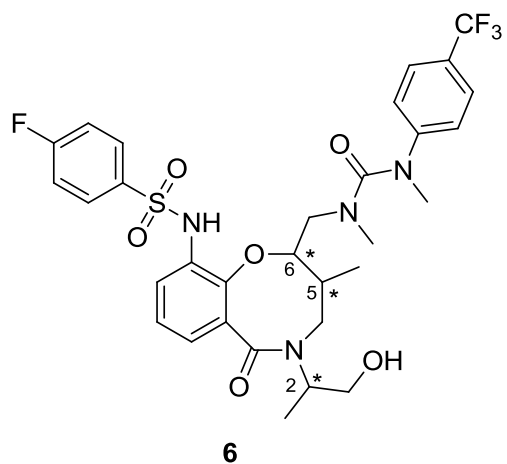

\begin{tabular}{|c|c|c|c|}
\hline RSS & RRS & RSR & RRR \\
\hline SSS & SRS & SSR & SRR \\
\hline
\end{tabular}

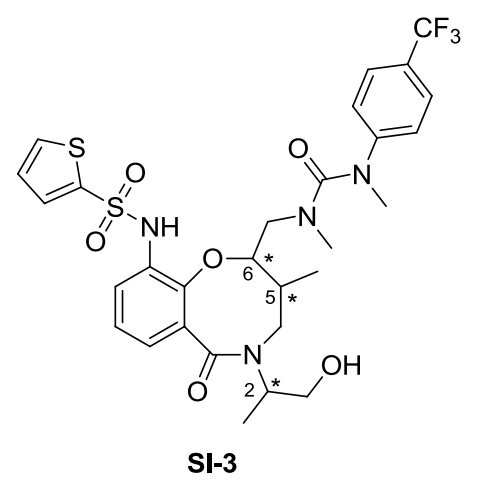

\begin{tabular}{|c|c|c|c|}
\hline RSS & RRS & RSR & RRR \\
\hline SSS & SRS & SSR & SRR \\
\hline
\end{tabular}

SI-3 
Figure SI-2: The lowest energy conformation of the free ligand 6 (green), 7 (orange), and the C2 epimer of $\mathbf{6}$ (magenta) are aligned on the macrocycle core in the crystal structure of $\mathbf{7}$ (yellow).

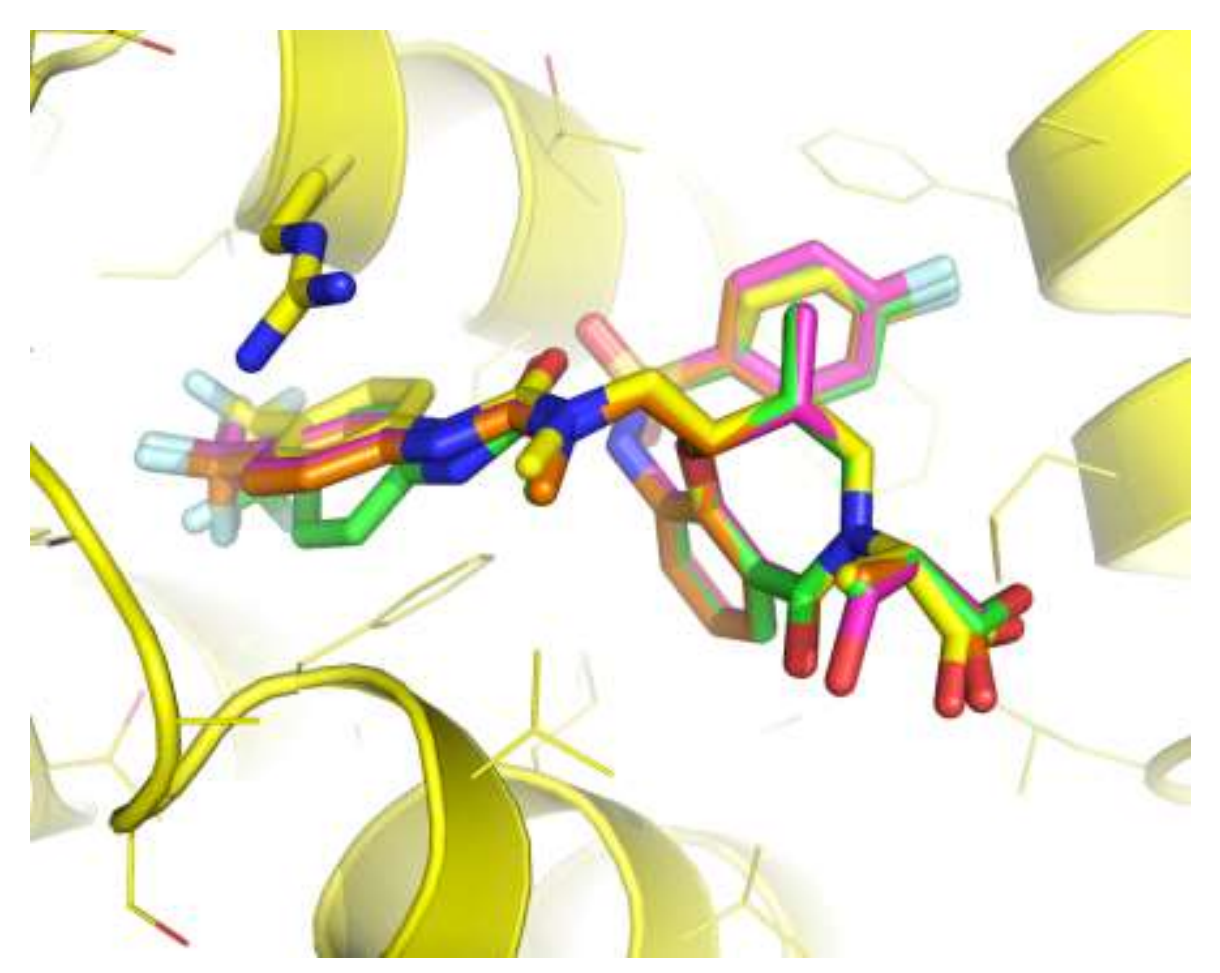

Conformations of compound 7, compound $\mathbf{6}$, and the exocyclic C2 stereoisomer of $\mathbf{6}$ were generated using the LowModeMD conformational search method in CCG MOE version 2013.08 using the MMFFs force field with Born solvation. The default parameters and charges were used for the force field. The conformational search maintained all conformations within $5 \mathrm{kcal} / \mathrm{mol}$ throughout the calculation and terminated after 100 consecutive steps with optimization led to conformers already visited. A cutoff of $0.25 \AA$ RMSD on all heavy atoms and energy determined conformer uniqueness. As shown in the figure, out of $100 \mathrm{~s}$ of conformations within $5 \mathrm{kcal} / \mathrm{mol}$, the lowest-energy conformations matched extremely well with the $1.8 \AA$ resolution crystal structure. 
Figure SI-3: DSC data for all eight isomers of 6

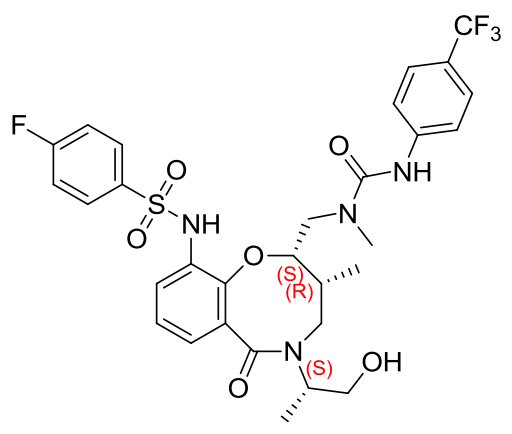

6

\begin{tabular}{c|cccccccc}
\hline $\mathrm{C}_{2} \mathrm{C}_{5} \mathrm{C}_{6}$ stereochemistry & SRS & RRS & SRR & RRR & SSR & RSR & SSS & RSS \\
\hline $\mathrm{DSC} \Delta \mathrm{T}_{\mathrm{m}}{ }^{\circ} \mathrm{C}$ & 1.9 & 2.1 & 0.3 & -0.1 & -0.2 & -0.3 & 0.0 & -0.1 \\
\hline
\end{tabular}


Figure SI-4: FP data of 7 against Bcl-2 and Bcl-xL

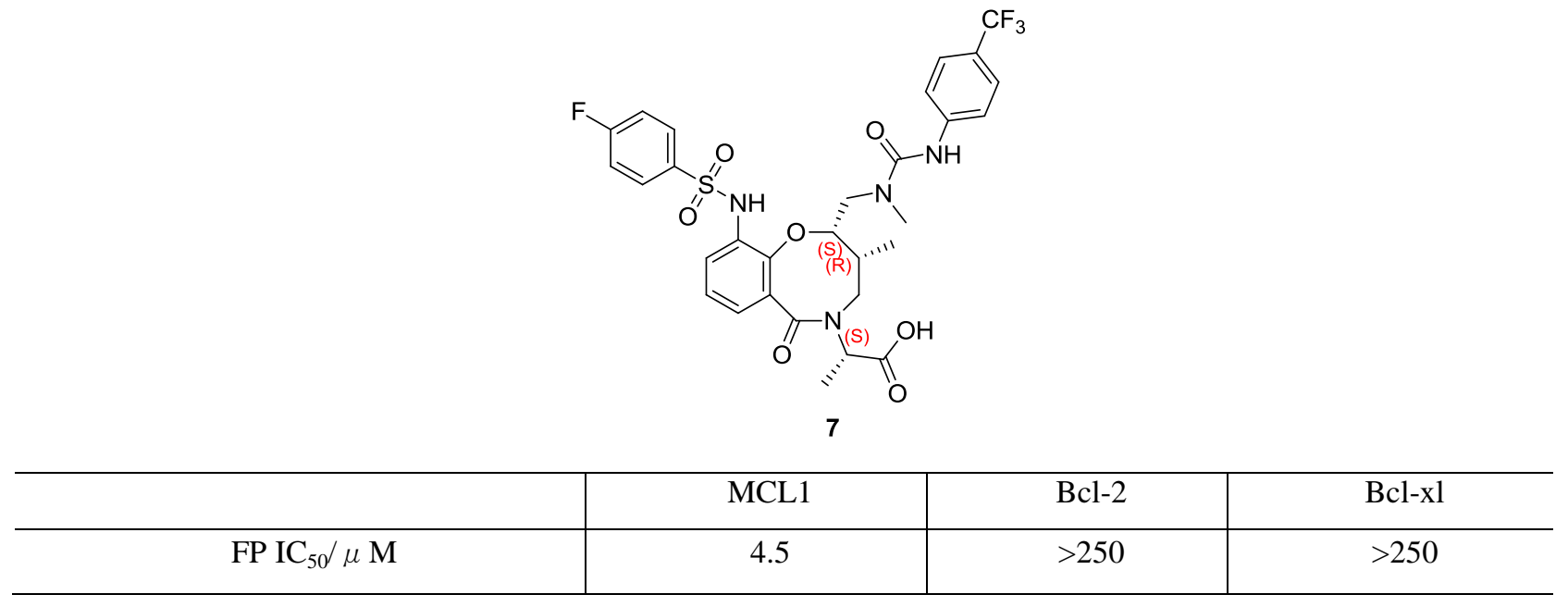


Figure SI-5: DSC and ITC data for fragments 19-20

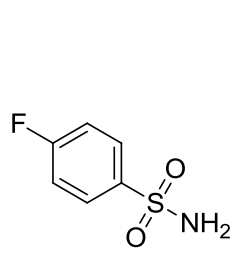

19

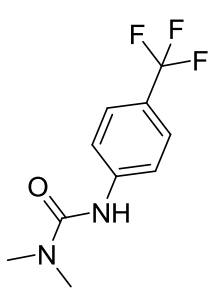

20

\begin{tabular}{c|c|c}
\hline compound & $\mathbf{1 9}$ & $\mathbf{2 0}$ \\
\hline $\mathrm{DSC} \Delta \mathrm{T}_{\mathrm{m}} /{ }^{\circ} \mathrm{C}$ & 0.1 & -0.3 \\
\hline ITC & No binding & No binding \\
\hline
\end{tabular}




\section{References:}

1. Marcaurelle, L. A.; Comer, E.; Dandapani, S.; Duvall, J. R.; Gerard, B.; Kesavan, S.; Lee, M. D.; Liu, H.; Lowe, J. T.; Marie, J.-C.; Mulrooney, C. A.; Pandya, B. A.; Rowley, A.; Ryba, T. D.; Suh, B.-C.; Wei, J.; Young, D. W.; Akella, L. B.; Ross, N. T.; Zhang, Y.-L.; Fass, D. M.; Reis, S. A.; Zhao, W.-N.; Haggarty, S. J.; Palmer, M.; Foley, M. A. J. Am. Chem. Soc. 2010, 132, 16962.

2. Kabsch, W. XDS. Acta Cryst. 2010, D66, 125.

3. McCoy, A. J.; Grosse-Kunstleve, R. W.; Adams, P. D.; Winn, M. D.; Storoni, L. C.; Read, R. J. J. Appl. Cryst. 2007, 40, 658.

4. Winn, M. D.; Ballarad, C. C.; Cowtan, K. D.; Dodson, E. J.; Emsley, P.; Evans, P. R.; Keegan, R. M.; Krissinel, E. B.; Leslie, A. G. W.; McCoy, A.; McNicholas, S. J.; Murshudov, G. N.; Pannu, N. S.; Potterton, E. A.; Powell, H. R.; Read, R. J.; Vagin, A.; Wilson, K. S. Acta. Cryst. 2011, D67, 235.

5. Afonine, P. V.; Grosse-Kunstleve, R. W.; Echols, N.; Headd, J. J.; Moriarty, N. W.; Mustyakimov, M.; Terwilliger, T. C.; Urzhumtsev, A.; Zwart, P. W.; Adams, P. D. Acta Crystallogr D Biol Crystallogr 2012, 68, 352.

6. Emsley, P.; Cowtan, K. Acta Crystallographica Section D-Biological Crystallography 2004, 60: 2126.

7. Chen, V. B.; Arendall, W. B.; Headd, J. J.; Keedy, D. A.; Immormino, R. M.; Kapral, G. J.; Murray, L. W.; Richardson, J. S.; Richardson, D. C. Acta Cryst. 2010, D66, 12. 